\title{
Cosmic Rays and Large Extra Dimensions
}

\author{
D. Kazanas \\ LHEA, NASA/GSFC Code 661, Greenbelt, MD 20771 \\ A. Nicolaidis \\ Department of Theoretical Physics, University of Thessaloniki, Thessaloniki \\ 54006, Greece
}

\begin{abstract}
We have proposed that the cosmic ray spectrum "knee", the steepening of the cosmic ray spectrum at energy $E \gtrsim 10^{15.5} \mathrm{eV}$, is due to "new physics", namely new interactions at $\mathrm{TeV} \mathrm{cm}$ energies which produce particles undetected by the experimental apparatus. In this letter we examine specifically the possibility that this interaction is low scale gravity. We consider that the graviton propagates, besides the usual four dimensions, into an additional $\delta$, compactified, large dimensions and we estimate the graviton production in $p p$ collisions in the high energy approximation where graviton emission is factorized. We find that the cross section for graviton production rises as fast as $\left(\sqrt{s} / M_{f}\right)^{2+\delta}$, where $M_{f}$ is the fundamental scale of gravity in $4+\delta$ dimensions, and that the distribution of radiating a fraction $y$ of the initial particle's energy into gravitational energy (which goes undetected) behaves as $\delta y^{\delta-1}$. The missing energy leads to an underestimate of the true energy and generates a break in the inferred cosmic ray spectrum (the "knee"). By fitting the cosmic ray spectrum data we deduce that the favorite values for the parameters of the theory are $M_{f} \sim 8 \mathrm{TeV}$ and $\delta=4$.
\end{abstract}

Key words:

PACS: 95.30Cq, 96.40De, 98.7Sa, 12.60.-i

Cosmic rays $(\mathrm{CR})$ is a subject almost a century old; despite the amount of knowledge accumulated since their discovery, there is a number of outstanding issues concerning their origin, acceleration and composition. One of the most interesting features is the breadth of their energy spectrum which extends over 11 orders of magnitude up to and beyond $10^{20} \mathrm{eV}$ (see [1] for a recent review).

The CR spectrum can be described as a broken power law $E^{-\gamma}$, with $\gamma$ obtaining three different values in three different energy regimes: In the range $10^{9}-10^{15.5}$

Preprint submitted to Elsevier Preprint 31 October 2018 
$\mathrm{eV}, \gamma \simeq 2.75$. At energies greater than $10^{15.5}$, the "knee", the spectrum steepens to $\gamma \simeq 3$ out to an energy $E \sim 10^{18} \mathrm{eV}$ (the "ankle"), at which point it becomes flatter again, $\gamma \simeq 2.2-2.5$ and extends to $\sim 10^{20.5} \mathrm{eV}$, beyond which the flux is too small to give a meaningful flux even with the largest current detectors.

The currently most popular models for cosmic ray acceleration (supernova shocks) can account for the $\mathrm{CR}$ spectra up to energies $10^{14} Z \mathrm{eV}$ only $(Z$ is the corresponding nuclear charge). Measuring the composition and understanding the origin of the cosmic ray spectrum above these energies remains a challenge both experimentally and theoretically. Of particular interest in this respect is the origin of the steepening of the CR spectrum at the "knee": As we suggested in earlier work [2], this (in fact any) spectral steepening cannot be accounted for by considering the spectrum to be the sum of two independent CR components, presumably the output of two different kinds of CR sources (an explanation that would be valid for a flattening of the spectrum such as that observed at the "ankle"). Furthermore, we suggested in the above reference that the simplest explanation of the CR spectrum break at the "knee" is that it is due to a novel channel in the physics of high energy $p p$-collisions, as proposed also by others in the past [3]. We also noted there that the $\mathrm{cm}$ energy corresponding to the "knee" is a few TeV, a scale tantalizingly close to the energy at which the emergence of "new physics" is anticipated on very general grounds. We advanced the idea that the new interaction, operative at and above these energies, results in particles that are not detected by the associated experimental devices, leading to an underestimate of the incident particle's energy for energies above that of the "knee". As a result, a cosmic ray spectrum which is a single power law in energy, will develop an increase in its slope (a "knee") at the energy at which the new interaction turns-on, with the spectrum reverting to its original slope when saturation occurs.

The main motivation for introducing new physics comes from the need to provide a unified theory in which two disparate scales, i.e. the electroweak $M_{W} \sim 100 \mathrm{GeV}$ and the Planck scale $M_{P} \sim 10^{19} \mathrm{GeV}$ can coexist (hierarchy problem). Recently a novel approach has been proposed for resolving the hierarchy problem [4]. Specifically, it has been suggested that our four dimensional world is embedded in a higher dimensional space with $D$ dimensions of which $\delta$ dimensions are compactified with a relatively large radius. While the Standard Model (SM) fields live on the 4-dimensional world (brane), the gravitons can propagate freely in the higher dimensional space (bulk). The fundamental scale $M_{f}$ of gravity in $D$ dimensions is related to the observed 4-dimensional Newton constant $G_{N}$ by

$$
G_{N}=\frac{1}{V_{\delta}}\left(\frac{1}{M_{f}}\right)^{(2+\delta)}
$$


where $V_{\delta}$ is the volume of the extra space. For a torus configuration

$$
V_{\delta}=(2 \pi R)^{\delta}
$$

with $R$ the common radius of the large extra dimensions. For a given $\delta$, a sufficiently large radius $R$ can then reduce the fundamental scale of gravity $M_{f}$ to $\mathrm{TeV}$ energies which are not too different from $M_{W}$, thereby resolving the hierarchy problem.

The $D$-dimensional graviton, when reduced to 4 -dimensions, gives rise to spin 2 particles (the massless graviton and its massive Kaluza-Klein excitations), spin 1 particles and spin zero particles. The vector particles do not couple to the energy momentum tensor and are ignored, while the scalar particles couple to the trace of the energy momentum tensor, giving negligible contributions to the high energy regime we are interested in $[5,6]$. Thus we take into account only the production of spin 2 gravitons in the $p p$-collisions we consider.

We study the $p p \rightarrow p p$ collision at the cm system, where each particle carries energy $\sqrt{s} / 2$. At high energies the dominant contribution to graviton $(h)$ production $(p p \rightarrow p p h)$, originates from collinear bremsstrahlung of gravitons from each external line. In this configuration, graviton emission is factorized as the probability that a proton (incoming or outgoing) loses a fraction $y$ of its energy via graviton radiation. The Kaluza-Klein (KK) excitations of the graviton have the same couplings to ordinary fields as their massless zeromode, i.e. they couple to the 4-momentum of the fermion they are attached. Within this reasonable approximation of factorized emission, we obtain that the cross-section for graviton production in $p p$ collisions, with the graviton carrying energy $\epsilon$, is given by

$$
\frac{d \sigma_{h}}{d \epsilon}(p p \rightarrow p p h)=\sigma_{0} \frac{4 G_{N}}{\pi} s \log \left(\frac{s}{m_{p}^{2}}\right) \frac{g(\epsilon)}{\epsilon}
$$

where $\sigma_{0}$ is the $p p \rightarrow p p$ cross-section (it rises slowly with energy), $m_{p}$ is the proton mass and $g(\epsilon)$ is the multiplicity factor counting the number of KK gravitons contributing to the process. The mass of each KK mode corresponds to the modulus of its momentum in the direction transverse to the brane

$$
m_{n}^{2}=\frac{\mathbf{n}^{2}}{R^{2}}
$$

with $\mathbf{n}=\left(n_{1}, n_{2}, \ldots, n_{\delta}\right)$. At fixed $\epsilon$, all gravitons with $m_{n} \leq \epsilon$ contribute, their number being the volume of a $\delta$-dimensional sphere with radius $n_{\max }=R \epsilon$. 
Thus

$$
g(\epsilon)=\frac{2 \pi^{\delta / 2}}{\delta \Gamma(\delta / 2)}(R \epsilon)^{\delta}
$$

Substituting expression (5) into eq. (3), using relation (1), absorbing constants and $\delta$-dependent factors into a rescaled $M_{f}$, we find that the cross-section for a fractional energy loss $y(y=2 \epsilon / \sqrt{s})$ is

$$
\frac{d \sigma_{h}}{d y}(p p \rightarrow p p h)=\sigma_{0} F(s) f(y)
$$

with

$$
\begin{gathered}
F(s)=\left(\frac{\sqrt{s}}{M_{f}}\right)^{2+\delta} \log \left(\frac{s}{m_{p}^{2}}\right) \\
f(y)=\delta y^{\delta-1}
\end{gathered}
$$

We observe that the cross-section for graviton emission rises fast, as a power of the energy (i.e. $\left.\propto\left(\sqrt{s} / M_{f}\right)^{2+\delta}\right)$ and while it is unimportant at energies below the scale $M_{f}$, it is the dominant process at and just above $M_{f}$. The distribution of the energy radiated into massless gravitons is represented by the well-known infrared $1 / y$ behavior, but the large number of KK modes converts this distribution into $y^{\delta-1}$. We emphasize again that within our approximative scheme we pick-up the leading contributions originating from collinear, soft, gravitational radiation. For the purposes of the present investigation we consider this approximation as well justified.

In the lab system a cosmic ray particle of high energy hits a nucleon at rest in the Earth's atmosphere. For a fractional energy loss $y$ in the $\mathrm{cm}$ system, in the lab system the corresponding event of total energy $E^{\prime}$ will be registered at the detector at an energy $E=E^{\prime}(1-y / 2)^{2}$. Therefore, for a cosmic ray intensity $I(E)$, gravitational radiation will induce an inferred intensity $N(E)$ of the form

$$
\left.N(E)=\int d E^{\prime} \int_{0}^{1} d y f(y) I\left(E^{\prime}\right) \delta\left(E-E^{\prime}(1-y / 2)^{2}\right)\right)
$$

The cosmic ray interactions will proceed either through the standard channels with probability $P_{0}(E)$, or through the production of gravitons with probability $P_{h}(E)$. We deduce that the observed cosmic ray intensity $I_{o b}(E)$ at an 


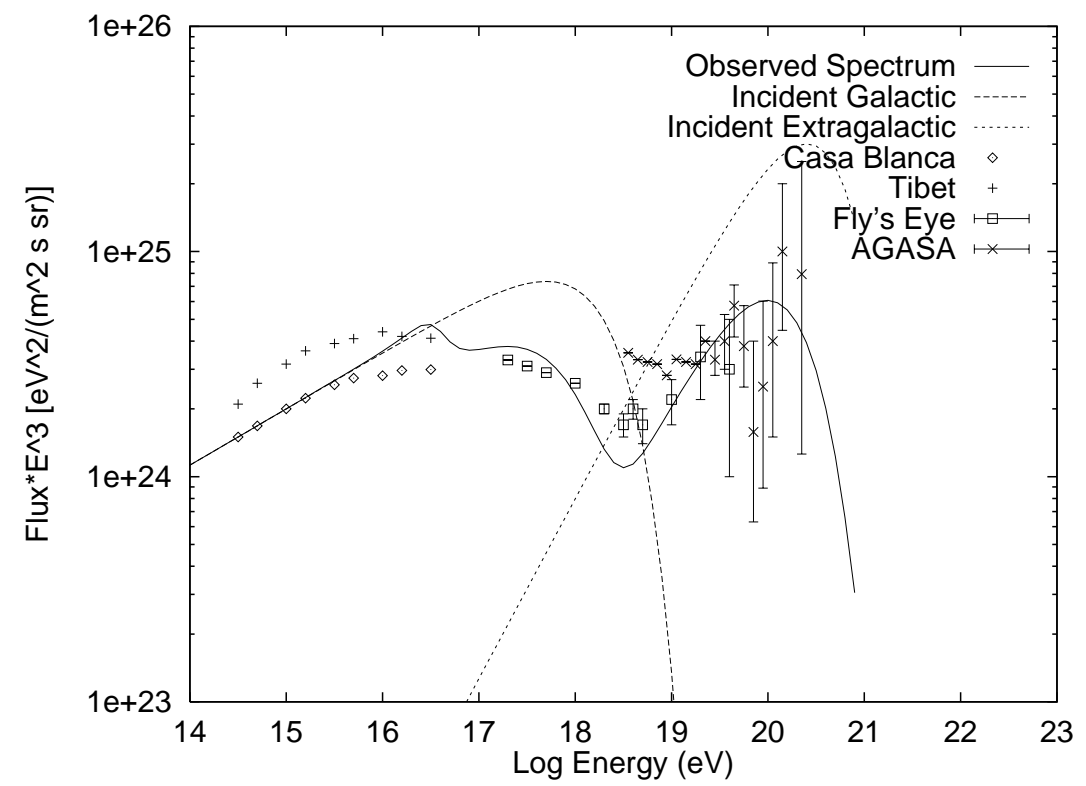

Fig. 1. The cosmic ray spectrum $f(E)$ multiplied by $E^{3}$ for $E>10^{14} \mathrm{eV}$. Long and short dashed lines are respectively the incident galactic and extragalactic components. Solid line is the combined spectrum expected to be measured for $M_{f}=8 \mathrm{TeV}, \delta=4 .+$ 's are the Tibet data, diamonds the Casa Blanca data, squares the Fly's Eye data and $\times$ 's the AGASA data.

energy $E$ will be the sum of these two processes, i.e.

$$
I_{o b}(E)=P_{0} I(E)+P_{h} N(E)
$$

where

$$
\begin{aligned}
& P_{0}(E)=\frac{\sigma_{0}}{\sigma_{0}+\sigma_{h}}=\frac{1}{1+F} \\
& P_{h}(E)=\frac{\sigma_{h}}{\sigma_{0}+\sigma_{h}}=\frac{F}{1+F}
\end{aligned}
$$

We assume that the incident cosmic ray spectrum $I(E)$ is composed of two components, the galactic component $\left(I_{G}(E)\right)$ and the extragalactic component $\left(I_{E}(E)\right)$ each of which is assumed to be a power law of indices $\gamma=$ 2.75 and 2.2 respectively, with the galactic component being dominant at energies $E \lesssim 10^{18.5} \mathrm{eV}$ and the extragalactic one at higher energies as discussed in [2] (i.e. $I_{G}(E) \propto E^{-2.75} \exp \left(-E / E_{0}\right), E_{0} \sim 10^{18.5} \mathrm{eV}$ and $I_{E}(E) \propto$ $\left.E^{-2.2} \exp \left(-E / E_{1}\right), E_{1} \sim 10^{20.5} \mathrm{eV}\right)$. According to our suggestion, the observed spectrum $I_{o b}(E)$ results from a migration of high energy points of energy $E^{\prime}$ to lower energy $E$ [see Eq. (9)]. Therefore, the inferred spectrum is a sensitive function of the input spectrum $I(E)$. Indeed the resulting spectrum, shown in fig. 1 , depends both on the parameters of the theory $\left(M_{f}, \delta\right)$ and the parame- 
ters of the input spectrum, notably the value of the galactic cut-off energy $E_{0}$. Rather than varying freely the parameters of the input spectrum, we have chosen the values adopted in our previous work [2] for the reasons explained there. The uncertainty in the experimental data, exemplified by the disagreement between two experiments in each energy range (i.e. Tibet - CASA BLANCA for $E=10^{14.5}-10^{16.5} \mathrm{eV}$, Fly's Eye - AGASA for $E>10^{17.5} \mathrm{eV}$ ) presented in figure 1, does not allow a high significance determination of the parameters of the theory. We found however that the data favor the values $M_{f} \simeq 8 \mathrm{TeV}$ and $\delta=4$ for the theoretical parameters, with the values $\delta=3,5$ being of lower significance as it was determined by the numerical study. Our deduced values respect all available bounds on $M_{f}$ and $\delta$. The most severe bounds come from the observations of SN 1987a, which constrain the rate of energy loss due to new particles [7]. Detailed caclulations [8] provide the limit $M_{f} \gtrsim 1 \mathrm{TeV}$ for $\delta=4$.

Cosmic accelerators accelerate particles (the cosmic rays) to energies much higher than those achieved at terrestrial laboratories. As such they present us with the opportunity of detecting the signatures of processes operative at energies not yet accessible at terrestrical accelerators. As mentioned in the beginning of the present note, the lowering of the fundamental Planck scale $M_{f}$ to $\mathrm{TeV}$ energies, implies that the gravitational interaction becomes strong at these energies. Any scattering process at $\mathrm{cm}$ energies $E \sim M_{f}$ should be accompanied by abundant graviton production. Since the energy of the cosmic rays can by far exceed this threshold domain, graviton emission should be preeminent in CR interactions. The essence of our proposal is that the signature of emission of these gravitons, which go undetected in the cosmic ray air shower arrays, is the observed "knee structure" in the CR spectrum.

We would like to conclude with a few general remarks. How reliable is our calculation? It is obvious that our cross section for graviton emission rises very fast and at some energy unitarity will be violated. The KK formulation we employed is an effective theory one and at some energy $M_{s}\left(M_{s}\right.$ is the string scale with $M_{s}>M_{f}$ ) we have to resort to the underlying theory, a string theory. With gravity dominant at $\mathrm{TeV}$ scales, gravitational radiation is only one of the possible manifestations. Exchange of virtual KK gravitons enhances the cross-sections [9] and this enhancement might help to resolve the paradox with the CR events above the GZK cut-off [10]. It has been suggested also that black holes may in fact be produced in $p p$ collisions [11]. Their subsequent decay through the Hawking radiation should lead to events of high multiplicity and large sphericity at the cm. It is of interest that detailed analysis of the EAS data suggest an apparent, very sharp change in the CR composition to almost exculsively Fe just above the "knee" (see fig. 5 of [12]). This apparent change is qualitatively of the form expected by a sharp increase in the interaction cross section and an ensuing dispersion of the available energy to a large number of secondary particles. The process described just 
above might then provide an account of this fact, though we do not believe that this interpretation can be considered at this point as unique. Finally, on the cosmic ray physics side, our proposal implies that the galactic CR sources produce power law spectra extending to the "ankle" rather than the "knee" (as thought on the basis of acceleration in SN shocks). Given that the latter do constitute CR sources to these energies our arguments imply the presence of two independent galactic CR components: one due to SN shocks, while clues of the origin of the second, being the observed anisotropy of CR at $E \sim 10^{18}$ $\mathrm{eV}$ toward the galactic center [13]. It appears that cosmic rays rather than being the messengers, constitute the message itself.

DK would like to thank Frank Jones and Bob Streitmatter for a number of discussions, comments, criticism and encouragement. AN would like to acknowlegde useful discussions with Ignatios Antoniadis and Costas Counnas. Part of this work was presented by AN at the Paris workshop on Physics and Astrophysics of Extra Dimensions (May 29 2001) and at the NESTOR Institute, Pylos (June 28 2001).

\section{References}

[1] T. K. Gaisser, astro-ph/0011524

[2] D. Kazanas and A. Nicolaidis, astro-ph/0103147

[3] S. I. Nikolsky, Bull. Russ. Acad. Sci. 57595 (1993)

[4] I. Antoniadis, Phys. Lett. B 246, 377 (1990); N. Arkani-Hamed, S. Dimopoulos, and G. Dvali, Phys. Lett. B 429, 263 (1998) I. Antoniadis, N. Arkani-Hamed, S. Dimopoulos and G. Dvali, Phys. Lett. B 436, 257 (1998)

[5] G. Giudice, R. Rattazzi and J. Wells, Nucl. Phys. B544, 3 (1999)

[6] T. Tan, J. Lykken and R. Zhang, Phys. Rev. D59, 105006-1 (1999)

[7] G. Raffelt, Stars as Laboratories for Fundamental Physics (University of Chicago Press, 1996)

[8] S. Cullen and M. Peerelstein, Phys. Rev. Lett. 83268 (1999); V Barger, T Han, C. Kao and R Zhang, Phys. Lett. B 46134 (1999); C. Hannart, D. Phillips, S. Reddy and M. Savage, Nucl. Phys. B595, 335 (2001); S. Hannestad and G. Raffelt, hep-th/0103201 preprint; D. Arndt, nucl-th/0102040 preprint

[9] S. Nussinov and R. Shrock, Phys. Rev. D59, 105002 (1999); C. Tyler, A. Olinto and G Sigl, Phys. Rev D63, 055001 (2001); P. Jain, D. McKay, S. Panda and J. Ralston, Phys. Lett. B484, 267 (2000); M. Kachelriess and M. Plümacher, Phys. Rev. D62, 103006 (2000) and hep-ph/0109184; F. Cornet, J. Illana and M. Masip, Phys. Rev. Lett. 86, 4235 (2001), also hep-th/0102065; J. AlvarezMuniz, F. Halzen, T. Han and D. Hooper, hep-ph/0107057; 
[10] K. Greissen, Phys. Rev. Lett. 16, 748 (1966); G.T. Zatsepin and V. A. Kuzmin, Pis'ma Zh. Eksp. Th. Fiz., 4, 114 (1966)[JETP Lett. 4, 78 (1966)]

[11] S. Dimopoulos and G. Landsberg, hep-th/0106295; S. Giddings and S. Thomas, hep-th/0106219

[12] J. W. Fowler et al., Astroparticle Physics, 15, 49; also astro-ph/0003190

[13] N. Hayashida et al. Astroparticle Physis 10303 (1999) 